\title{
Development and novel design of clustery Graphene Oxide formed Conductive Silk hydrogel cell vesicle to Repair Myocardial Infarction: Investigation of its biological activity for cell delivery applications
}

Rui Li ( $\sim$ lrui55@yahoo.com )

Huaihe Hospital of Henan University https://orcid.org/0000-0001-6917-4613

$\mathrm{Na}$ Duan

Central Hospital of Kaifeng City

Jingjing Guo

Huaihe Hospital of Henan University

\section{Research}

Keywords: Myocardial infarction, Silk Protein, Graphene oxide, Progenitor cells, Growth factors

Posted Date: June 18th, 2020

DOI: https://doi.org/10.21203/rs.3.rs-36186/v1

License: (c) (i) This work is licensed under a Creative Commons Attribution 4.0 International License.

Read Full License 


\section{Abstract}

Cell delivery therapy is a talented and hopeful strategic approach to repair the damaged cardiac tissues after myocardial infarction. The biocompatible injectable hydrogel with bioactive nanomaterials with effective self-healing capability has been highly required for the cell delivery and regeneration therapy. In the present study, we have developed the biopolymeric and biocompatible self-healing silk protein hydrogel matrix with graphene oxide nanoformulations as cell delivery vehicles for the treatment of myocardial infarction regeneration. The materials combinations, structural interactions, thermal stability and morphology of the designed hydrogel were investigated and elaborated. To improve therapeutic potential of the hydrogel matrix, growth factor was loaded and investigated its biological activity such as cell survival and cell proliferations properties by using endothelial progenitor cells. Collected, these developed materials are excellent vesicle for cell therapy in myocardial infarction.

\section{Introduction}

Recent years, heart failures after myocardial infarction is one of the primary causes of human mortality worldwide, and creating a therapeutic challenge for biomedical researchers[1,2]. The native myocardial tissue is a main part of transferring electrical signal and influencing factor of heart beat functions, while myocardial infarction leads to unwanted scar formations of myocardial tissues[3-5]. The damage of myocardium is chief causes of mechanical and electrical signal loss, which leads to weakened cardiac functions and consequently made heart failures. To get regenerative functions of these impaired myocardial functions, cell therapy has occurred as a promising strategy from the results of recent researches and clinical trials[6-8]. The cardiac activated cells are typically delivered through intracoronary delivery system; though, recent meta-analysis reports exhibited a limited outcome on cardiac regenerations and myocardial recoveries because of survival loss and apoptosis of delivered cells. These cell deaths resulted in the antagonistic biological environment at the damaged myocardial tissues[6-12]. To improve the therapeutic efficiency of cell therapy in myocardial recovery, there several strategies have been approached to enhance the cell survival rate through delivering cells under carriers of angiogenic growth factors, genetically altered stem cells, and importantly biocompatible functional biomaterials such as nanomaterials, patches, hydrogels, etc.,[11, 13-15]. Particularly, favorable cell delivery via injectable hydrogel system can be a stimulating and effective alternative to attain the targeted site delivery of huge numbered live cells. Many biodegradable hydrogels designed containing a broad range of favorable mechanical and biocompatible properties have been investigated by clinical animal models to regeneration of myocardial infarction, cardiovascular diseases, etc., Numerous reported studies were suggested that high modulus injectable hydrogel matrix with conductive properties could be greatly influence on recovery of heart functions after myocardial infarction[16-19].

The combination of injectable bio-hydrogel matrix with biocompatible and electro-active nanomaterials that provide promising activity and cell retention in the cardiac therapies[20]. The fabrications of ideal hydrogel scaffolds could confirm the optimistic cellular activity to support cell proliferations, which is greatly favorable as a stem cell carrier. Additionally, the loading of nanomaterials into the hydrogel have 
been induce electric transmission, inter and intra-cellular communications and particularly metabolic activity at micro-environment of myocardial infarcted site[21-23]. In addition, the improving mechanical properties and micro-environment of hydrogel matrix provides favorable inhibitions ability against ventricular remodeling in MI[24, 25]. Silk protein is a widely available natural bio-polymer derived from silk cocoons that has unique biological and mechanical abilities, which provides effective actions in regenerative medicine, drug delivery, bio-adhesives, etc.,[3, 6, 23]. The biocompatibility, mechanical stability, biodegradability properties of silk protein hydrogels can be modulate for the required microenvironmental conditions[3, 23]. Specifically, the cardiovascular disease therapies and myocardial regenerations are greatly depended on the electrical conductivity of the implanted materials for cellular functions co-ordinations and signal transductions of electroactive tissues[5, 26]. Hence, recent studies about cardiac repair have preferred that introduction of electroactive components (e.g., conductive polymers, conductive nanoformulations, etc.,) to implanted substrates could prominently develop neuronal cellular behaviors in clinical trials. In this present investigation, modified graphene oxide nanomaterial has incorporated into the SF hydrogel combined with stem cells could enhance the cardiac repair efficiency.

\section{Materials And Methods \\ 2.1. Materials}

Bombyx mori silkwarm cocoons was obtained from local silk production company, Suzhou, China. Graphene oxide (GO) and Carbodiimide cross-linking agents (EDC-NHS) were purchased from Beijing Sinopharm Chemical Reagent company Ltd., China. Other analytical grade solvents and reagents were acquired from Sigma Aldrich., USA, and used without any additional purification methods.

\subsection{Preparation of Aqueous SF solution}

The regenerated aqueous Silk fibroin solution was prepared under the protocols of previously published reports. In brief, cocoons of Bombyx silkwarm were boiled in water with addition of $\mathrm{Na}_{2} \mathrm{CO}_{3}(0.05 \mathrm{wt} \%)$ for 60 min to remove the residual sericin proteins. Then, it was rinsed thoroughly by using deionized water and after that dried for $12 \mathrm{~h}$ in hot air oven at $60^{\circ} \mathrm{C}$. The purified silk fibroin fibrous sponges were dissolved into a freshly prepared highly concentrated Lithium Bromide ( $\mathrm{LiBr} ; 9.3 \mathrm{M})$ solution at $60^{\circ} \mathrm{C}$ for 3 hours and then dialyzed (cellulose dialysis membrane) for 3 days under DI water to remove residual salts. Finally, the dialyzed solution was centrifuged to eliminate agglomerated and non-soluble residues and then regenerated aqueous SF solution was obtained.

\subsection{Preparation of $\mathrm{GO}$ solution and NHS/EDC activated $\mathrm{GO}$}

The commercially acquired GO nano-powder was used to prepare GO substrate solution by using water medium. The $\mathrm{GO}(0.25 \mathrm{mg} / \mathrm{mL}$ and $0.5 \mathrm{mg} / \mathrm{mL})$ powder was dispersed in DI water separately under highfrequency ultrasonication for $2 \mathrm{~h}$ to reduce formation of agglomerated particles. The prepared solution was then filtered using polycarbonate filter papers $(5.0 \mu \mathrm{m})$ to eliminate bulk particles from the solution. 
Simultaneously, the solution of EDC/NHS was prepared by using freshly made $0.01 \mathrm{M}$ PBS medium ( $\mathrm{pH}$ = 7.4) under magnetic stirring and kept into ultrasonic machine for $10 \mathrm{~min}$. Then, the prepared NHS/EDC medium was mixed with the above prepared GO solution $(1 \mathrm{mg} / \mathrm{mL})$ to activate carboxylic group of graphene oxide surfaces. The mixed medium was allowed to react GO with NHS/EDC at $4{ }^{\circ} \mathrm{C}$ for $24 \mathrm{~h}$.

\subsection{Preparation of $\mathrm{GO} / \mathrm{SF}$ blended hydrogel}

The Graphene Oxide loaded SF hydrogel was prepared by using the above prepared carboxylic activated GO solution and regenerated SF with addition of PEG as a gelation agent. In brief, two different concentrations of activated-GO $(0.25$ and $0.5 \mathrm{mg} / \mathrm{mL})$ solution was added into the SF solution and PEG solution to placed for stirring under sonication for $1 \mathrm{~h}$. The obtained injectable GO/SF hydrogel samples were stored at $-20^{\circ} \mathrm{C}$ for further biological investigations. The prepared samples were primarily frozen and lyophilized at $-80^{\circ} \mathrm{C}$ for $48 \mathrm{~h}$ for the physico-chemical characterizations. The schematic representations of the present work has been exhibited in Fig. 1.

\subsection{Characterization of prepared materials}

The chemical structure and interactions between the $\mathrm{GO}$ and fibroin molecules were studied under Fourier transform spectroscope (Nicolet iN10; Thermofisher, USA) and Raman spectroscopic (Renishaw) techniques. The surface morphology and micrographic structures of $\mathrm{mGO}$ loaded SF was recorded and visualized by scanning electron microscope (Hitachi 3000-N) at $10 \mathrm{kV}$ and atomic force microscopic (Veeco Nanoscope IV system, CA) imaging technique at a scanning speed $1.001 \mathrm{~Hz}$. The samples for SEM and AFM were prepared by dissolution of $\mathrm{mGO} / \mathrm{SF}$ in aqueous medium and coated on copper plate and fresh mica, respectively. The sample were dried well at room temperature before microscopic observations.

\subsection{Isolation of cardiac progenitor cell (CPCs)}

Cardiac progenitor cells were isolated from the removed heart tissues of sacrificed Sprague-Dawley rats, as followed animal experimental protocols of Institutional animal Care and Use committee, China. The standardized heart tissue was further processed with collagenase (type-2; solubilized with HBSS solution) and was filtered through a $70 \mu \mathrm{m}$ filter. The isolated cells were sensibly incubated with dynabeads conjugated c-kit antibody after magnetic preceding to a magnetic sorting. The organized cells by sorting were cultured into a tissue culture place and prolonged to cellular confluence. Following that, the CPCs viable properties were determined by flow cytometric analysis of c-kit, multi-drug resistance protein, Gata4 and Nkx2.5. The isolated cells have the cloning ability above $90 \%$ were only used for the further analysis and cell implantations into the hydrogel matrix.

\subsection{Implantation of CPCs into the hydrogel matrix}

The previously prepared GO/SF hydrogel was sterilized and it made for the suitable cell culture conditions by using freshly prepared phosphate buffered saline (PBS) medium. Then, the hydrogels were standardized by the cell culture medium (DMEM; GlutaMax-l; Anti-biotics) and incubated for $1 \mathrm{~h}$. This 
process was repeated by two time to get standard hydrogel for the cell survival. After that, appropriate quantity of isolated CPCs was directly pipetted onto the hydrogel in an appropriate cell growth conditions to provide strength for implant.

\subsection{In vitro cell survival analysis}

The CPCs cell compatibility and survival analysis into the prepared hydrogel samples were determined as already described protocol. After implantation of CPCs into the hydrogels, the Live/Dead viability assay was used to analyze the cell viability percentage as followed by the manufacturer's instructions. The hydrogel treated cells were displayed by using fluorescent microscope (Leica, Germany) and analyzed using LASAF version 2.0.2 software.

\section{Result And Discussions}

The spectral analysis of FTIR and Raman techniques have been extensively used to investigate chemical structure, functionalization's and functional groups interactions of carbon-based nanomaterials. The Raman spectral data of GO was displayed the characteristic peaks at the range of $1340 \mathrm{~cm}-1$ and $1594 \mathrm{~cm}-1$ as exhibited in Fig. 2 (b), corresponding to the bands of D and G, respectively. Generally, the intensity values of Raman bands (ID and IG) specifies the oxidation degree of carbon-materials and/or $\mathrm{sp} 3 / \mathrm{sp} 2$ carbon ratio of graphene oxide. The presented results indicated that intensity value of $D$ band has been increased significantly after oxidation, which designates the ID/IG ratio was influenced and existed the higher oxidation degree of GO. The enhanced oxidation degree and oxygen functional groups of GO could greatly favorable for the formation hydrogen bonding between the carboxylic groups and $\mathrm{NH}_{2}$ groups of silk protein molecules, which confirms the strong interfacial avenue and chemical interactions of components.

To analyze the structural confirmation of silk protein molecules after incorporation of GO, the prepared matrix was investigated through FTIR spectroscopy (Fig. 2b). The characteristic peaks of silk proteins have been observed at the ranges of $1620 \mathrm{~cm}-1$ (amide I), $1515 \mathrm{~cm}-1$ (amide II) and $1230 \mathrm{~cm}-1$ (amide III), which corresponded to the $\beta$-sheet formation in presented composites and bare SF. In addition, disappearance of the peak at $1665 \mathrm{~cm}^{-1}$ of all SF composited forms, specifying that the alteration of chemical composition from random coil to formation of $\beta$-sheet. These annotations of FTIR spectral analysis are confirmed the effective alteration of SF from water soluble form to non-soluble form. The results of FTIR and Raman spectral analyses have been specified the strong associations between nanoformulations of RGO and molecular chains of silk protein structure. In brief, the distribution of GO nanostructures into the SF-hydrogel materials could be influencing the formation of silk II crystal structures into final SF/GO matrix product. This is happening due to the presence of hydrogen bonding formations and creation of intermolecular forces between the components. In addition, the incorporation of GO into the SF matrix has been influenced to the rearrangements of SF adjacent chain segments after formation of intermolecular interactions and hydrophilic or hydrophobic interactions, which is leading to the new bond's formation in the new positions. 
The thermal stability of the composite materials was investigated under TGA analysis method and results of the presented matrix and bare components exhibited in Fig. 3 (A). The prepared SF/RGO nanocomposite have losing weights in three different temperatures ranges. Firstly, weight loss observed within $100^{\circ} \mathrm{C}$, due to the water absorption. And maximum weight loss of the prepared materials has been detected in the range of $260^{\circ} \mathrm{C}$ and $380^{\circ} \mathrm{C}$, which is associated with char residue formation of SF compounds. Final stage involves to thermal degradation of char after $380^{\circ} \mathrm{C}$. The results of thermal stability analysis demonstrated that prepared RGO/SF nanocomposited matrix have greater stability than bare SF matrix, due to the incorporation of GO nanoformulations. The analysis data of XRD have demonstrated the uniform distribution of RGO nanoformulations into SF matrix with the respective increasing intensity peaks of silk II structures as exhibited in Fig. 3(B). The characteristics XRD pattern peaks were observed at $9.7,20.7$ and $25.2^{\circ}$, which confirmed to the formation's silk II crystal structure after incorporation of RGO. In addition, the observation of XRD data indicated that direct interactions between the RGO nanoformulations and crystal structure of silk II of silk protein matrix. The surface morphological structure of the prepared hydrogels was visualized by FE-SEM images as shown in Fig. 4. The SEM observation was clearly demonstrated that fabricated hydrogels have greater and uniform porous structural morphology, which could be favorable for the cell attachment and bioactivity in the cardiac regenerations. In addition, we have observed SF hydrogel without GO nanoformulations had higher porous diameter compared to hydrogel with GO nanoparticles. This result of morphology analysis has confirmed the incorporation $\mathrm{GO}$ is influencing the morphological nature, and uniform and smaller porous structure may have greater effect to sustained delivery of growth factors and cell implantations.

After physico-chemical characterization of materials, the bioactivity and suitability of the hydrogel matrices were analyzed in vitro methods. The previous biological examinations exhibited that the prepared SF/GO hydrogels have an improved cytocompatibility, which could enhance the cell proliferations of endothelial cells and vascular cells. Then, cardio-regeneration ability of hydrogels was investigated by in vitro culture of cardiomyocytes onto the surface of prepared SF/GO hydrogels in different time intervals $(6,12$ and $24 \mathrm{~h}$ ) as displayed in Fig. 5 (a). The results of cardiomyocytes adhesion on hydrogel materials demonstrated that adhesion rate has been increased during increasing incubation period. At $24 \mathrm{~h}$, the number of cardiomyocytes on the SF/GO hydrogel surface is greatly increased and very higher than that on surface of bare SF hydrogel. In addition, the adhesion ability of cardiomyocytes on SF/GO and bare SF hydrogels has no significant changes, which confirms that cardiomyocyte has no any toxicity with silk-based hydrogels. Additionally, we have examined the cardiomyocytes survival rate with the treatment of prepared hydrogels. The survival rate of cardiomyocytes was tested in different incubation periods (1, 3 and 7 days). In addition, we have examined these SF matrices in the presence and absence of $\mathrm{GO}$ on the early cardiac transcription factors with cardiomyocyte progenitor cells by in vitro method. The results from expressions of early cardiac markers (SAC, Cx43 and cTnl) was have significant increase on the prepared SF matrices after 4 days of in vitro culture as exhibited in Fig. 6 . We could be confirmed that prepared biological SF matrices have efficient for early stage cardiac progenitor cells differentiation, which significantly associates with previous studies. 
The oxidative stress reproductive ability of the growth factor loaded SF hydrogel matrix in the presence and absence of GO was examined on the human coronary artery endothelial cells (hCAECs), which is induced by using $\mathrm{H}_{2} \mathrm{O}_{2}$. As shown in Figure ( $7 \mathrm{a}$ and $\left.\mathrm{b}\right)$, the live/dead analysis exhibited greater live cells on the surface of SF/GO hydrogel compared to bare SF hydrogels, which imply that incorporation GO nanoformulations have no toxic to cell survival and also provided greater compatibility for endothelial cells survival. The mentioned cells were cultured on the prepared hydrogel matrices for different days and the cell survival and proliferations were quantitatively observed as exhibited in Fig. 7. In previous studies have been elaborated that silk fibroin hydrogel supports cellular infiltrations and favorable proliferation properties with different cell types. In the present investigation, we have observed that GO nanoformulations incorporated SF hydrogel have enhanced cellular behaviors compared to the bare SF hydrogel matrix. The cell proliferations of GO/SF have no significant changes at days 1 and 3 , but the proliferation percentage has led to greatly enhanced for day 7 as exhibited in Fig. 5 (a).

The effect of SF/GO hydrogels on endothelial cells oxidative damage was analyzed by induction of $\mathrm{H}_{2} \mathrm{O}_{2}$. Generally, $\mathrm{H}_{2} \mathrm{O}_{2}$ is an important factor to persuading oxidative stress and damages on healthy cells to determination of in vitro model oxidative stress analysis. The previous reports established that cell survival was decreased with increasing concentration of $\mathrm{H}_{2} \mathrm{O}_{2}$, due to its inducing oxidative stress effect on cells. In the present investigation, $\mathrm{H}_{2} \mathrm{O}_{2}$ was used to induce cardiomyocytes oxidative stress and then incubated with prepared hydrogel to examine the protective ability against oxidative stress injury. The result of confocal microscopic images (Fig. 7) exhibited that SF/GO hydrogels have been improved the survival rate of the endothelial cells. Furthermore, the results clearly showed that number of live cells (green cells) in SF/GO hydrogels have significantly higher than that of SF hydrogel. After that we have examined another radical-related parameter of NO protection from the cardiomyocytes. Nitric oxide is a short time-lived free radical factors and essential inflammatory factors which has been contributed in activated cell apoptosis and greatly participate in numerous biological functions. As shown in Fig. 7 (c), the $\mathrm{NO}$ level of $\mathrm{CMs}$ induced by $\mathrm{H}_{2} \mathrm{O}_{2}$ has been greatly controlled and sustainably reduced by the addition of prepared hydrogels compared to the control sample. From the detailed investigations of the prepared hydrogel with encapsulation of growth factors, we found that CPC cells have great compatibility and survival rate into the developed hydrogels, which demonstrated that surface structure and mechanical abilities have been favorable for the regeneration of myocardial infarction. The biological investigations of cardiomyocytes survival and oxidative stress investigations clearly established that hydrogels with growth factor had great capability to cardiac cells differentiation and reproductive ability against ROS species. Our future work aims to apply this developed hydrogel loading with CPC to achieve efficient myocardial infarction regeneration and higher cardiac cells differentiation by alteration of surface structure and mechanical properties. In addition, it is also very stimulating factors for analyses of different cell types (e.g., embryonic and pluripotent stem cells) encapsulation with optimized cryogel materials.

\section{Conclusion}


In the present investigation we have developed a nanoformulated silk protein hydrogels with encapsulation of growth factor with optimized porous surface structure and mechanical gelation properties to improve therapeutic potential of myocardial infarction regeneration. The morphology, chemical structure of GO loaded SF hydrogel was investigated successfully and confirmed the favorable observation regarded for the cardiac regeneration applications. The in vitro biocompatibility analysis on CPC was established that prepared hydrogels with growth factors have no negative stimulations and improved the cell survival rate, which confirms that prepared gel formulations with GO greatly favorable for the implantations for cardiac therapy. In addition, the oxidative stress and ROS re-protective analyses have demonstrated that prepared gel material positive influence and outstanding cardiomyocytes differentiations. The injectable SF hydrogels with tunable mechanical strength could be a hopeful cell delivery vesicle for myocardial infarction regeneration therapy.

\section{Declarations}

\section{Conflict of Interest}

Authors have no conflict of Interest

\section{Compliance with Ethical Standards}

No animal studies were carried out by the authors for this article.

\section{References}

1. T. Yoshizumi, Y. Zhu, H. Jiang, A. D’Amore, H. Sakaguchi, J. Tchao, K. Tobita, and W. R. Wagner, Biomaterials 83, 182 (2016).

2. Y. Matsumura, Y. Zhu, H. Jiang, A. D’Amore, S. K. Luketich, V. Charwat, T. Yoshizumi, H. Sato, B. Yang, T. Uchibori, K. E. Healy, and W. R. Wagner, Biomaterials 217, 119289 (2019).

3. N. H. Chi, M. C. Yang, T. W. Chung, N. K. Chou, and S. S. Wang, Carbohydr. Polym. 92, 591 (2013).

4. R. Bao, B. Tan, S. Liang, N. Zhang, W. Wang, and W. Liu, Biomaterials 122, 63 (2017).

5. W. Wang, B. Tan, J. Chen, R. Bao, X. Zhang, S. Liang, Y. Shang, W. Liang, Y. Cui, G. Fan, H. Jia, and W. Liu, Biomaterials 160, 69 (2018).

6. N. H. Chi, M. C. Yang, T. W. Chung, J. Y. Chen, N. K. Chou, and S. S. Wang, Biomaterials 33, 5541 (2012).

7. T. Yoshizumi, Y. Zhu, H. Jiang, A. D. Amore, H. Sakaguchi, J. Tchao, K. Tobita, and W. R. Wagner, Biomaterials 83, 182 (2016).

8. W. Wang, B. Tan, J. Chen, R. Bao, X. Zhang, S. Liang, and W. Liu, Biomaterials 160, 69 (2018).

9. X. Yao, Y. Liu, J. Gao, L. Yang, D. Mao, C. Stefanitsch, Y. Li, J. Zhang, L. Ou, D. Kong, Q. Zhao, and Z. Li, Biomaterials 60, 130 (2015). 
10. L. Ye, W. Zhang, L. P. Su, H. K. Haider, K. K. Poh, M. J. Galupo, G. Songco, R. W. Ge, H. C. Tan, and E. K. W. Sim, Biomaterials 32, 2424 (2011).

11. Y. Kambe and T. Yamaoka, Biomater. Sci. (2019).doi:10.1039/c9bm00556k

12. H. Wang, J. Shi, Y. Wang, Y. Yin, L. Wang, J. Liu, Z. Liu, C. Duan, P. Zhu, and C. Wang, Biomaterials 35, 3986 (2014).

13. K. Kadner, S. Dobner, T. Franz, D. Bezuidenhout, M. S. Sirry, P. Zilla, and N. H. Davies, 33, 2060 (2012).

14. H. Li, J. Gao, Y. Shang, Y. Hua, M. Ye, and Z. Yang, 1.

15. W. Wan, X. Jiang, X. Li, C. Zhang, X. Yi, S. Ren, and X. Zhang, 3452 (2013).doi:10.1002/jbm.a.35014

16. A. Junnan, T. M. D. Ph, X. C. P. D, and T. G. Caranasos, 1.

17. H. Li, J. Gao, Y. Shang, Y. Hua, M. Ye, Z. Yang, and C. Ou, ACS Appl. Mater. Interfaces 10, 24459 (2018).

18. Z. Wang, D. W. Long, Y. Huang, S. Khor, X. Li, X. Jian, and Y. Wang, (2017).doi:10.1021/acsbiomaterials.6b00509

19. C. Fan, J. Shi, Y. Zhuang, L. Zhang, L. Huang, W. Yang, and B. Chen, 1902900, 1 (2019).

20. Y. K. Wu and J. Yu, J. Mater. Chem. B 3, 6401 (2015).

21. H. E. M. Mewhort, J. D. Turnbull, A. Satriano, K. Chow, J. A. Flewitt, A. C. Andrei, D. G. Guzzardi, D. A. Svystonyuk, J. A. White, and P. W. M. Fedak, J. Hear. Lung Transplant. 35, 661 (2016).

22. D. H. Kim, Kshitiz, R. R. Smith, P. Kim, E. H. Ahn, H. N. Kim, E. Marbán, K. Y. Suh, and A. Levchenko, Integr. Biol. (United Kingdom) 4, 1019 (2012).

23. J. Chen, Y. Zhan, Y. Wang, D. Han, B. Tao, Z. Luo, S. Ma, Q. Wang, X. Li, L. Fan, C. Li, H. Deng, and F. Cao, Acta Biomater. 80, 154 (2018).

24. W. Liang, J. Chen, L. Li, M. Li, X. Wei, B. Tan, Y. Shang, G. Fan, W. Wang, and W. Liu, ACS Appl. Mater. Interfaces 11, 14619 (2019).

25. E. Ruvinov, J. Leor, and S. Cohen, Biomaterials 32, 565 (2011).

26. A. Navaei, N. Moore, R. T. Sullivan, D. Truong, R. Q. Migrino, and M. Nikkhah, RSC Adv. 7, 3302 (2017).

\section{Figures}




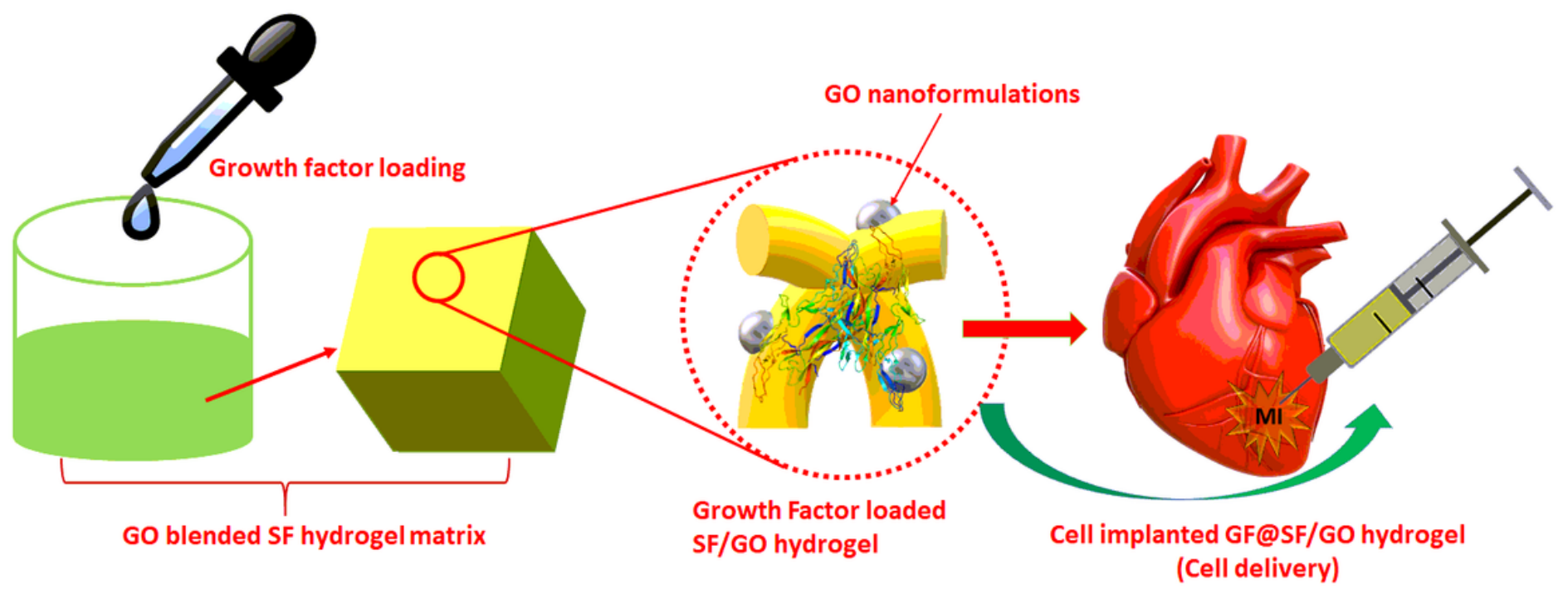

Figure 1

Schematic representation of the present investigation exhibits proposed formation structure of hydrogel network with GO nanoformulations and growth factors and showed schematic applied site of MI.
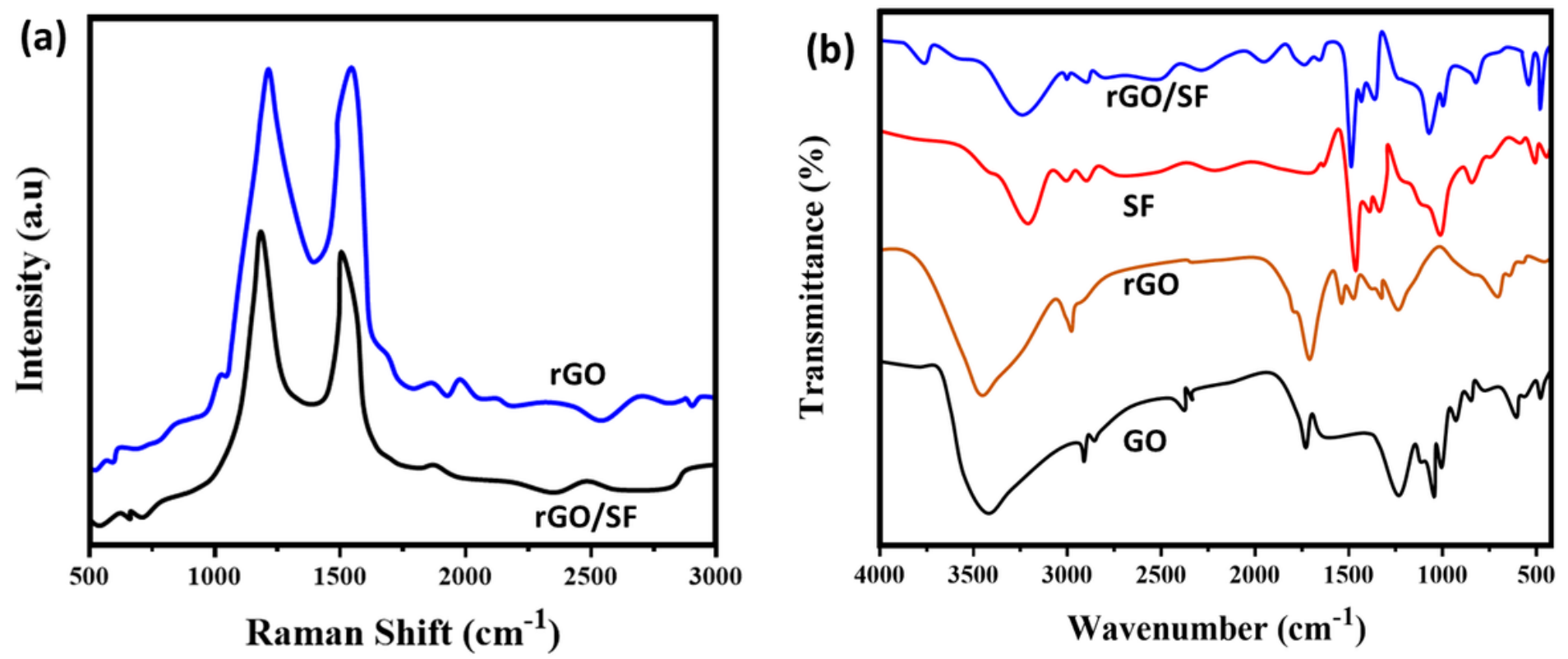

Figure 2

The chemical structure and interactions examinations of prepared samples: (a) Raman spectroscopic and (b) FTIR spectroscopic analyses observation exhibited for hydrogen and bare samples as mentioned in the visualizations. 

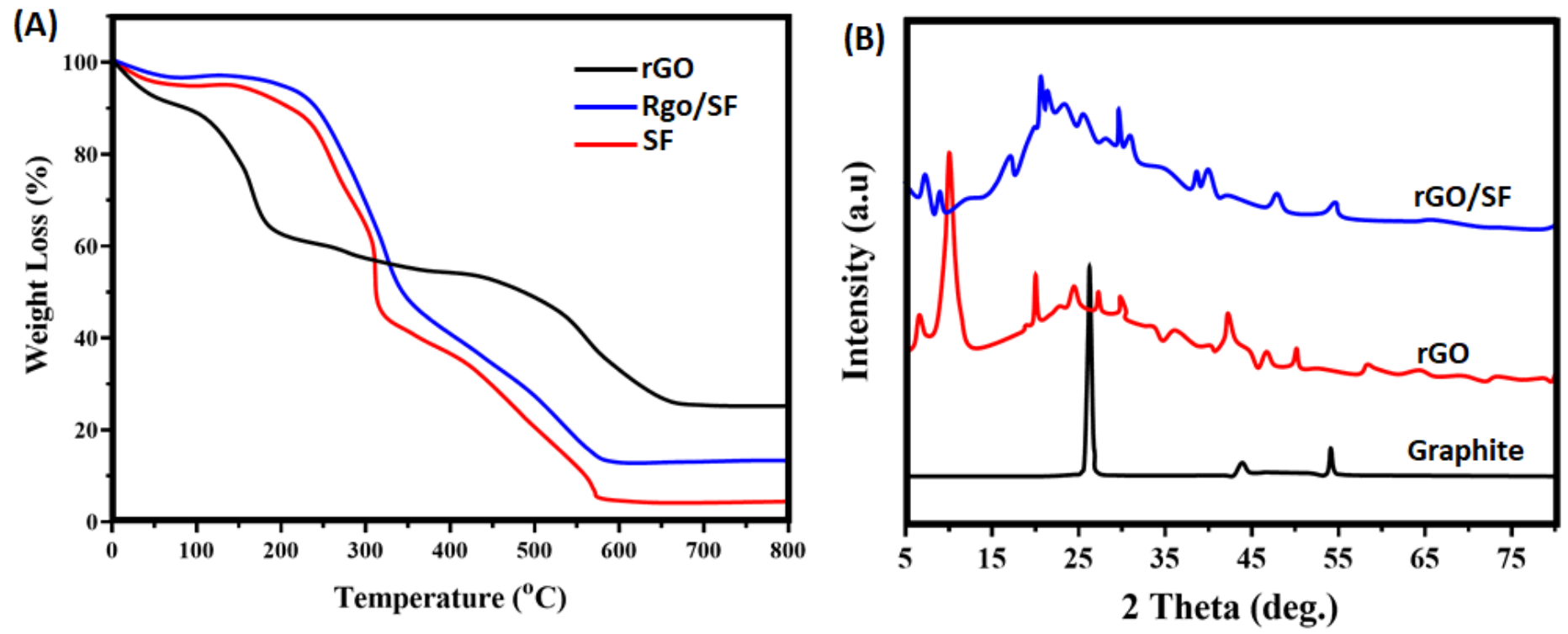

Figure 3

The thermal stability and phase purity of prepared samples: (A) TGA analysis and XRD pattern analysis results of the respective bare and hydrogel samples, which exhibited the changes of thermal and crystallinity behaviors. 

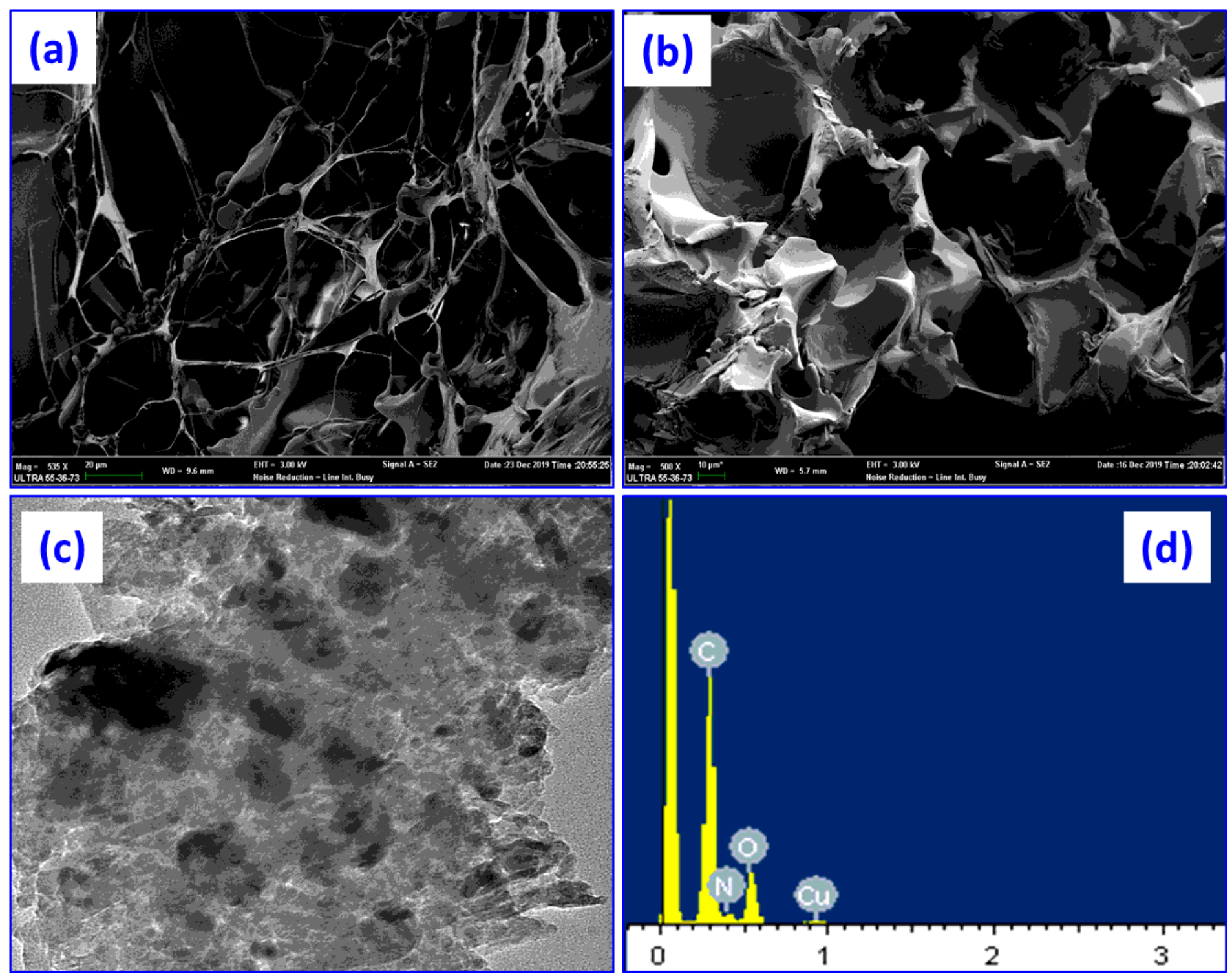

Figure 4

Surface morphological analysis: SEM analysis of SF hydrogel in the absence and presence of GO nanoclusters ( $a \& b)$, TEM observations of SF/GO hydrogel samples, which showed distributions of GO structure into the hydrogel structure and EDX analysis of SF/GO hydrogel. 

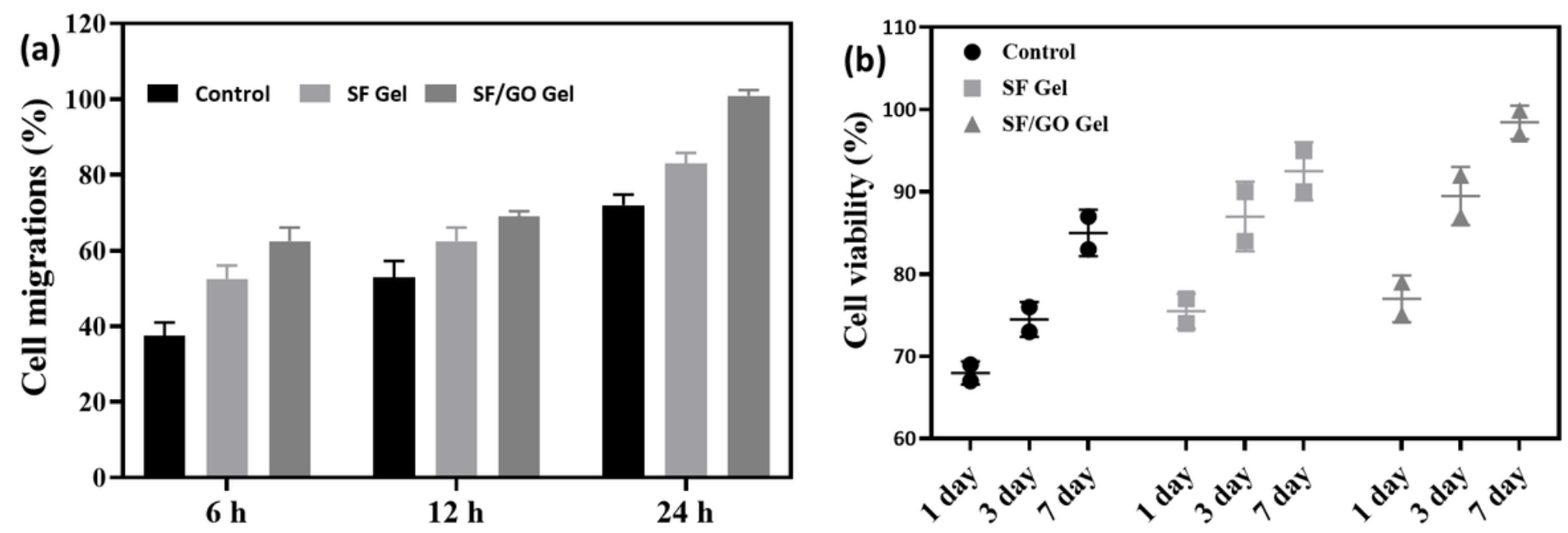

Figure 5

In vitro cell cytocompatibility analysis of prepared hydrogel samples: (a) cell proliferations in different incubation period and (b) cell viability percentages in 1, 3 and 7 days.

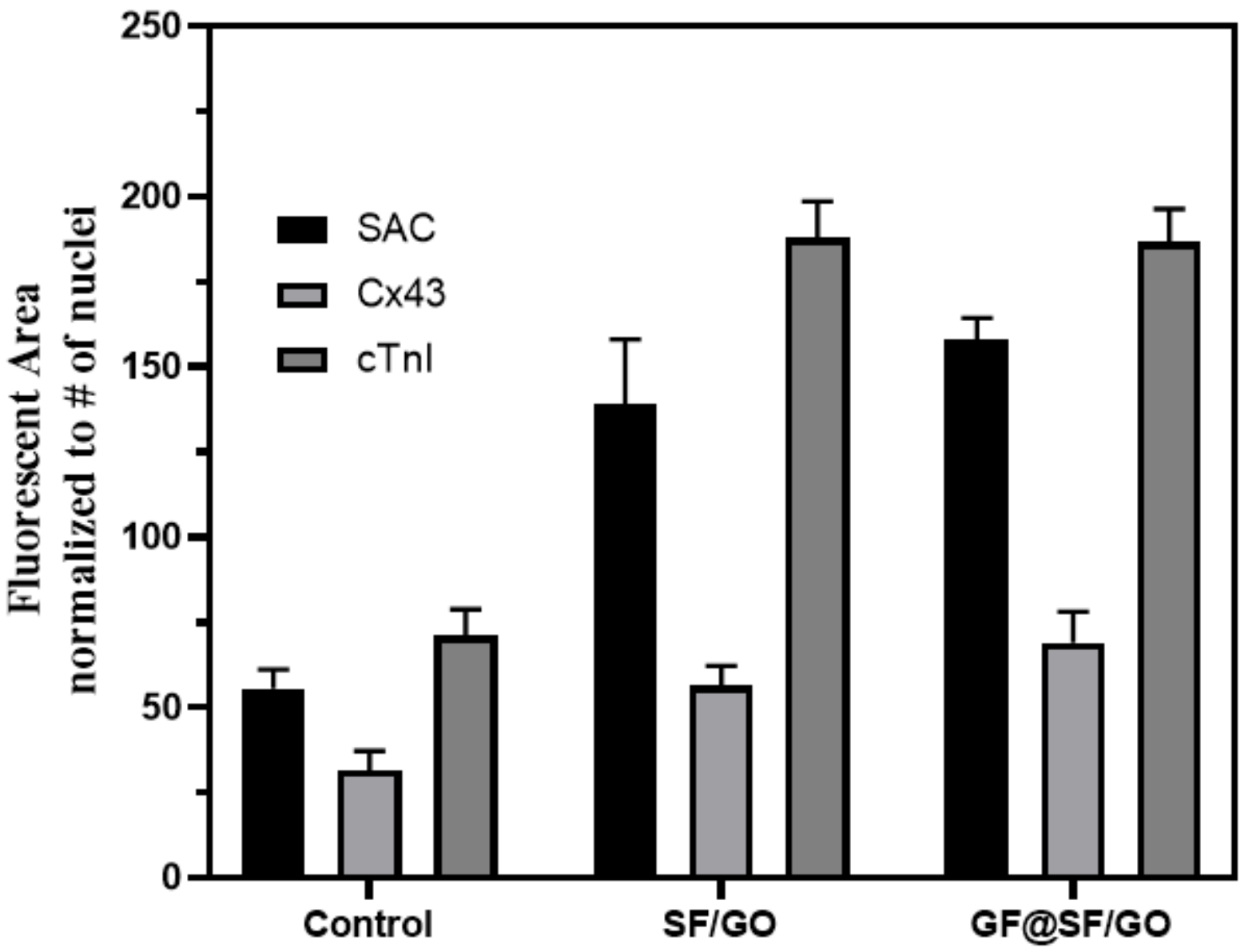

Figure 6 
In vitro examinations of early stage cardiac markers expressions by using cardiac progenitors' cells with prepared GF loaded SF/GO hydrogel samples.
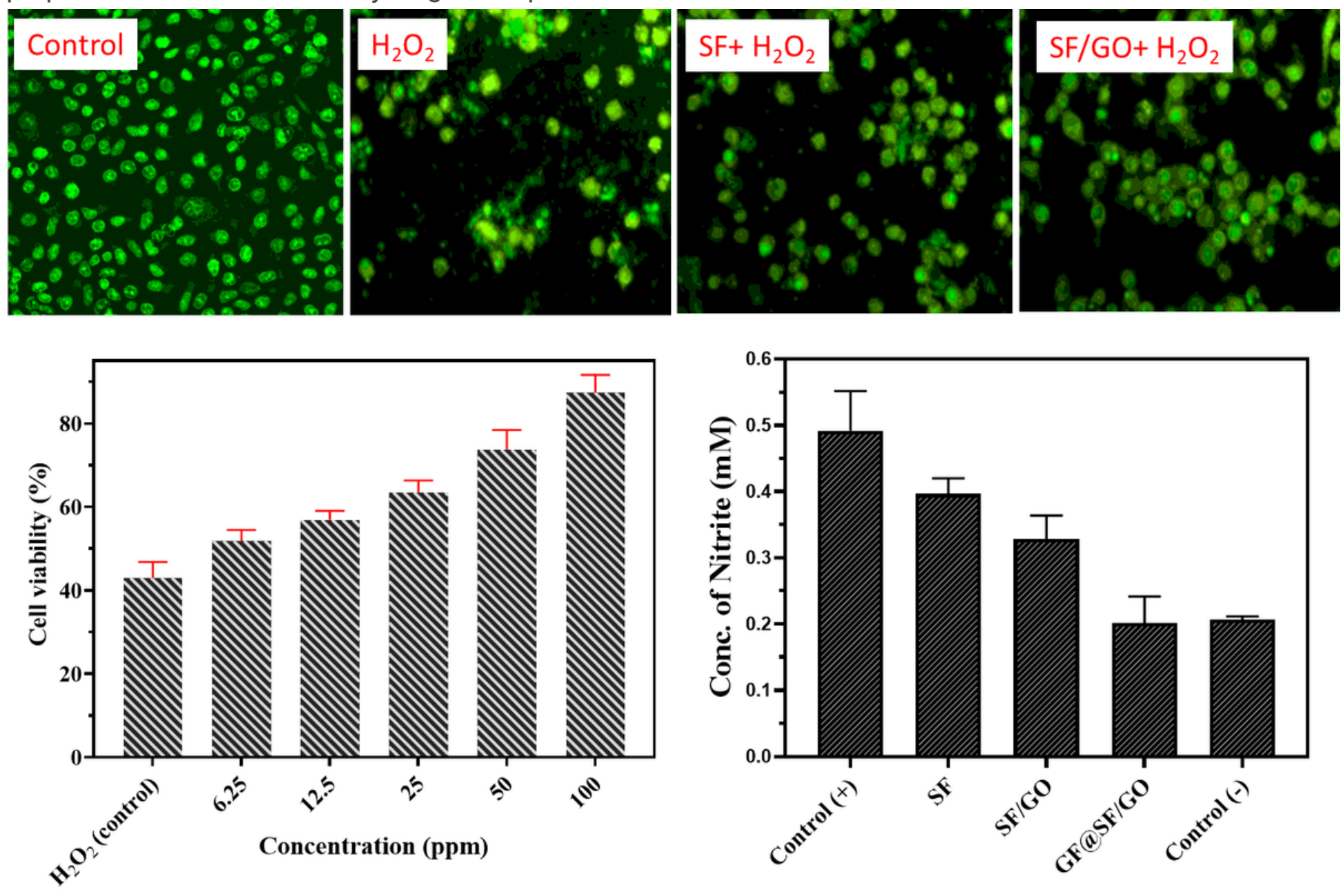

Figure 7

In vitro analysis of oxidative stress reproductive ability of the prepared samples induced by $\mathrm{H} 2 \mathrm{O} 2$.

\section{Supplementary Files}

This is a list of supplementary files associated with this preprint. Click to download.

- OnlineGraphicalabstract.Png 